\title{
Disponibilidad del recurso hídrico en la microcuenca del río Bermúdez. Región Central de Costa Rica
}

\author{
Ligia HERNANDO ECHEVARRÍA \\ Escuela de Ciencias Geográficas \\ Universidad Nacional de Costa Rica \\ ligia.hernando.echevarria@una.cr \\ Ricardo OROZCO MONTOYA \\ Escuela de Ciencias Geográficas \\ Universidad Nacional de Costa Rica \\ Riorozco1707@gmail.com
}

Recibido: 27 de agosto del 2015

Enviado a evaluar: 2 de septiembre del 2015

Aceptado: 25 de noviembre del 2015

\section{RESUMEN}

La microcuenca del río Bermúdez es parte de la principal zona de explotación hídrica en la región Central de Costa Rica, razón por la cual se realiza un diagnóstico de la disponibilidad del recurso hídrico en esta microcuenca donde se identifican las áreas con mayor problemática de disponibilidad de este recurso. Para ello se calculó un balance hídrico mensual, según uso del suelo, unidad geomorfológica y zona climática. Con base en este balance se determinó y clasificó la disponibilidad del recurso, identificando en la microcuenca solamente tres categorías: alta, media y moderada. No existen áreas de baja disponibilidad de recurso hídrico lo que demuestra que la oferta es suficiente, sin embargo, existe una presión importante sobre el recurso hídrico pues más de la mitad del área de la microcuenca se encuentra con una disponibilidad moderada.

Palabras clave: Balance hídrico, cuenca hidrográfica, disponibilidad del recurso hídrico.

\section{Availability of water resources in the río Bermúdez micro-basin. Central Region of Costa Rica}

\begin{abstract}
The Rio Bermúdez micro-basin makes up part of the principal hydrological resource area in the Central Region of Costa Rica. For this reason a study was done to determine the availability of hydrological resources in said micro-basin to identify areas with potential water availability problems. A monthly water balance was calculated using land use, geomorphology and climate parameters. From these water balance studies, the amount of available water was calculated and classified into four categories, however, in this micro-basin, only three categories were identified: high, medium and moderate water availability. No areas were identified with low water availability, indicating availability is sufficient;
\end{abstract}


however, there is increasing demand on water resources because over half of the micro-basin area is classified as having moderate water availability.

Key words: Water balance, watershed basin, water resource availability.

\section{Disponibilité de la ressource hydrique du microbassin fluviale du fleuve Bermúdez. Région Centrale du Costa Rica}

\section{RÉSUMÉ}

Le microbassin fluvial du fleuve Bermúdez fait parti de la principale zone d'exploitation en eau de la région centrale du Costa Rica. Afin de faire perdurer cette ressource, on effectue un diagnostique en disponibilité hydrique des aires les plus sensibles au tarissement. Il est fait pour cela un calcul du bilan hydrique mensuel regroupant les paramètres climatiques, d'utilisation du sol et la géomorphologie. Avec la base de données obtenue, on détermine et on classifie cette disponibilité en trois catégories : haute, moyen et modérée. N'existant pas d'aire de basse disponibilité, on peut supposer d'une offre suffisante de la ressource. À noter cependant que le bassin ayant plus de la moitié de son aire rentrant dans la catégorie «modérée », la pression anthropique sur la ressource hydrique est non négligeable.

Mots clés: Bilan hydrique, bassin hydrographique, disponibilité hydrique.

\section{INTRODUCCIÓN}

La gestión del recurso hídrico en Costa Rica se torna cada vez más difícil pues en muchos sectores su cantidad y distribución ha variado. En los últimos años en la Región Central de Costa Rica, las actividades industriales y el crecimiento urbano han aumentado notablemente, lo que trajo como consecuencia una mayor presión para este recurso. Esta presión provoca a su vez la necesidad de lograr un aprovechamiento adecuado del mismo, sustentando su disponibilidad bajo estas nuevas condiciones. Un insumo muy importante es la zonificación espacio-temporal de la disponibilidad del recurso hídrico, la cual debe realizarse con base en un balance hídrico, cuyos resultados se introducen en un Sistema de Información Geográfica (SIG) para realizar el análisis espacial correspondiente y obtener la zonificación deseada.

La Escuela de Ciencias Geográficas de la Universidad Nacional no se ha mantenido al margen de esta problemática, por lo que en las décadas de 1980 y 1990 se efectuaron algunos balances hídricos a nivel de Costa Rica, con muy buenos resultados. Actualmente se retoma esta línea de trabajo dada la necesidad expresada por instituciones a nivel regional de continuar este tipo de investigaciones. Desde el año 2000 este grupo de trabajo se encuentra realizando investigaciones en dicho campo, en las microcuencas ubicadas en el sector norte de la Región Central de Costa Rica, abarcando hasta el momento, las de los ríos Poás, Ciruelas, Segundo y ahora Bermúdez. Se pretende que durante los próximos cinco años sean abarcadas también las de los ríos Tibás y Pará. Con ello se busca brindar un aporte fundamental a nivel nacional que funcione para la toma de decisiones en el ámbito del uso adecuado del 
recurso hídrico, ya que se contará con información clara acerca de la distribución del mismo.

El principal objetivo de este estudio es realizar un diagnóstico de la disponibilidad del recurso hídrico en la microcuenca de Río Bermúdez, identificando las áreas de mayor problemática, dicha microcuenca está localizada en una zona de alto potencial hídrico, pues ésta abastece a la población de los principales centros urbanos de la región central de Costa Rica.

\section{2. ÁREA DE ESTUDIO}

La microcuenca se encuentra aproximadamente entre las coordenadas métricas 493.078m-1114.704m, 476.432m - 1101.448m y 492.468m - 1104.605m, así mismo

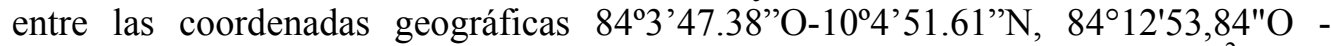
$9^{\circ} 57^{\prime} 39,90^{\prime \prime N}$, y $84^{\circ} 4^{\prime} 7,32^{\prime \prime O}-9^{\circ} 59^{\prime} 22,89 " \mathrm{~N}$; con un área total $74,11 \mathrm{Km}^{2}$. Está conformada por nueve cantones, de los cuales ocho son de la provincia de Heredia, y uno de la provincia de Alajuela (Figura 1).

Figura 1. Área de estudio.

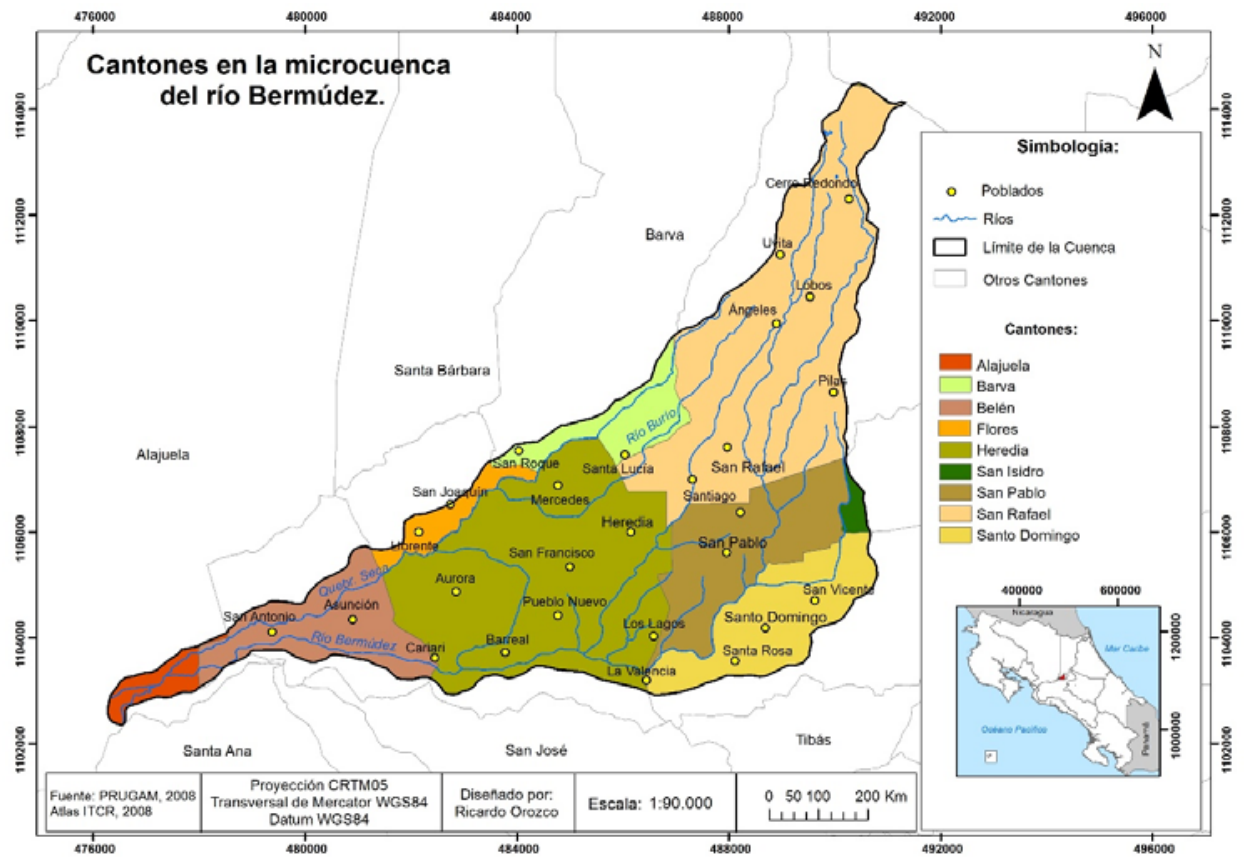

Fuente: Elaboración propia. 


\section{ASPECTOS GENERALES DE LA MICROCUENCA}

\subsection{GEOMORFOLOGÍA}

Según el mapa geomorfológico, escala 1:50.000 de Bergoeing, 1982, en el área se encuentran dos unidades geomorfológicas, una de origen volcánico y otra de origen sedimentario del Pleistoceno (Figura 2).

Figura 2. Unidades geomorfológicas.

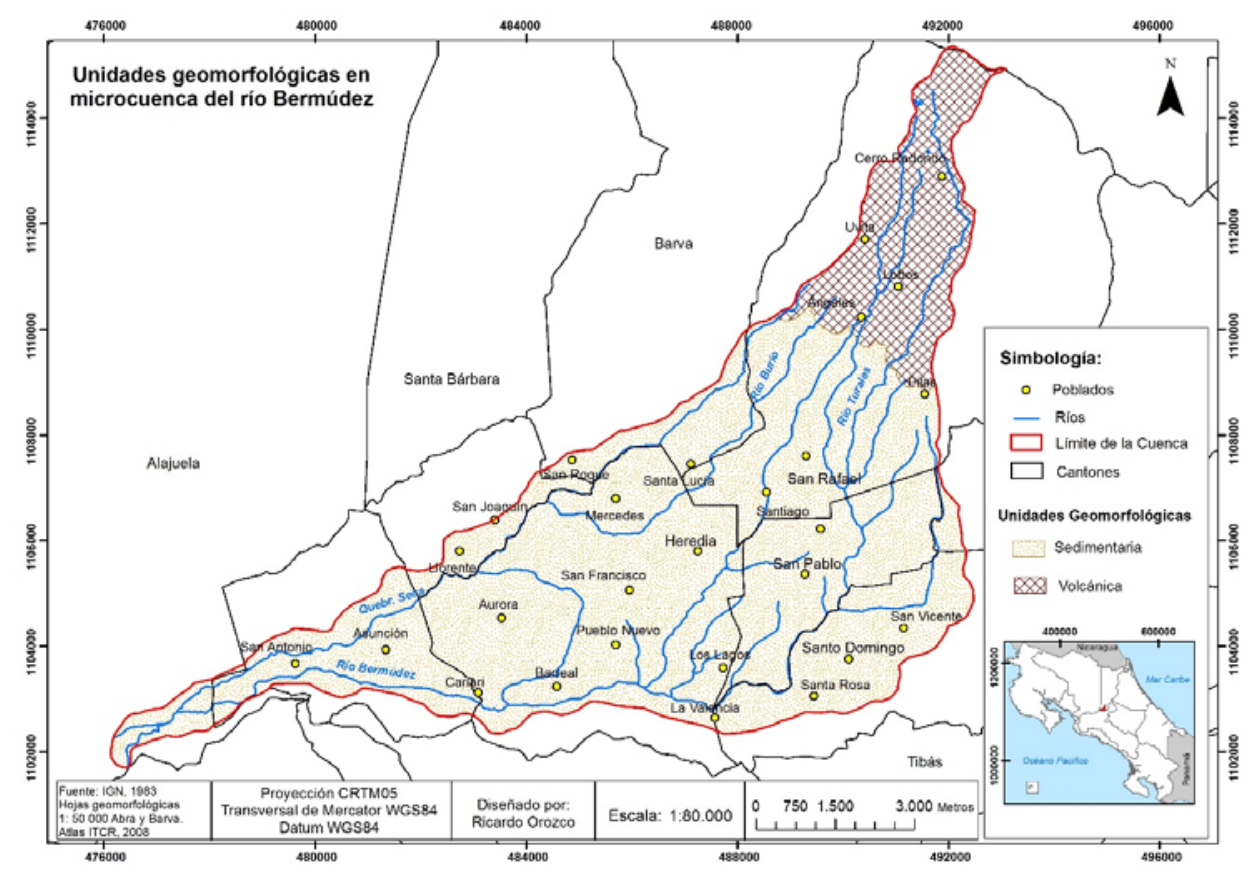

Fuente: Elaboración propia.

En la parte alta de la cuenca se presentan modelados fluviales tales como: cañones fluviales que corresponden al entallamiento profundo y súbito de un cauce fluvial que forman paredes verticales y dejan escarpes rocosos a la vista (Bergoeing, 1982). Estos los encontramos en el río Turales.

En la parte media sobre los $1500 \mathrm{msnm}$ y la parte baja cerca de los $900 \mathrm{msnm}$ se encuentran procesos erosivos tales como taludes de erosión, estos son sectores de vertientes que por su pendiente son inestables y por ello sujetas a intensa erosión.

En la unidad geomorfológica volcánica se encuentran frentes de colada de lava a partir de los $1500 \mathrm{msnm}$. Los niveles de terrazas se encuentran distribuidos desde la parte baja de la microcuenca hasta la parte media $(1700 \mathrm{msnm})$. 
La unidad geomorfológica sedimentaria se encuentra a partir de los $1500 \mathrm{msnm}$ y hasta los $1200 \mathrm{msnm}$. Se encuentran procesos sedimentarios de origen volcánico como depósitos de piedemonte. Aquí se encuentran poblados como Barva, Palmar, Paso viga, y San Rafael. Esta unidad geomorfológica está compuesta en su totalidad por lahares. Desde San Rafael hasta el final de la microcuenca se encuentran Planezes ${ }^{1}$.

\subsection{ZONAS CLIMÁTICAS}

Se describen a continuación cuatro zonas climáticas determinadas según el índice hídrico establecido por Thornthwaite (Hernando, L, 1988). Ver Figura 3.

Figura 3. Zonas climáticas.

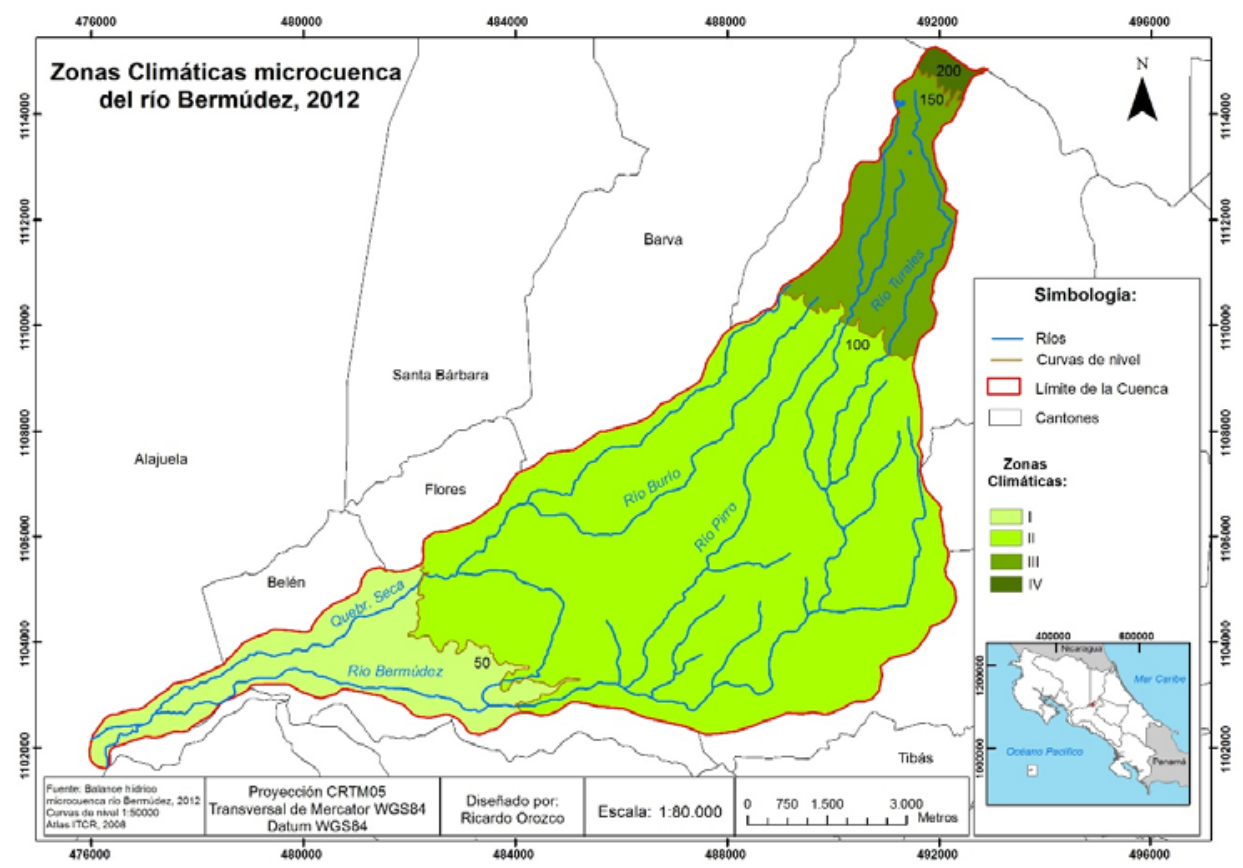

Fuente: Elaboración propia.

\footnotetext{
${ }^{1}$ Planezes son las formas derivadas de una erosión diferencial al quedar protegidos determinados relieves -conos y, especialmente, formaciones amesetadas- por coladas posteriores, resultando el edificio disectado en bloques de planta triangular. (Araña, V \& López, J, 1974).
} 
Zona Climática I, comprende $11 \mathrm{Km}^{2}$, posee un índice hídrico de hasta el $50 \%$ con una altitud máxima de $1000 \mathrm{msnm}$, de acuerdo a estas características su precipitación media anual es de $1957,7 \mathrm{~mm}$. Considerando las temperaturas que oscilan de $17.1^{\circ} \mathrm{C}$ a $27.7^{\circ} \mathrm{C}$, en esta área la evapotranspiración potencial media anual es de $1589.02 \mathrm{~mm}$.

Zona Climática II, es la zona que posee mayor cobertura en área, la cual tiene una cobertura de $54 \mathrm{Km}^{2}$, y posee un índice hídrico de $100 \%$ definido por las altitudes de $1000 \mathrm{msnm}$ a $1500 \mathrm{msnm}$. Por las condiciones que expresan esta zona, su precipitación es de $2415.62 \mathrm{~mm}$, y su evapotranspiración potencial media anual es de $1445.18 \mathrm{~mm}$ debido a que su temperatura varía de $15.6^{\circ} \mathrm{C}$ a $26.08^{\circ} \mathrm{C}$.

Zona Climática III, se caracteriza por poseer un área de $9 \mathrm{Km}^{2}$, la misma presenta una precipitación de $3028.52 \mathrm{~mm}$ aunado a una evapotranspiración potencial media anual de $1324.37 \mathrm{~mm}$, debido a que su temperatura varía de $12.9^{\circ} \mathrm{C}$ a $23.2^{\circ} \mathrm{C}$, estas características son representantes de una zona la cual se encuentra entre los 1500 msnm y $2000 \mathrm{msnm}$ con un índice hídrico de $150 \%$.

Zona Climática IV, comprende un área de $1 \mathrm{Km}^{2}$, esta zona recibe $3028.52 \mathrm{~mm}$ de precipitación media anual, y se presentan $1324.37 \mathrm{~mm}$ de evapotranspiración potencial media anual, esto debido a que esta zona se encuentra a más de $2500 \mathrm{msnm}$. Se caracteriza por presentar una temperatura entre $21.1^{\circ} \mathrm{C}$ y $10.9^{\circ} \mathrm{C}$ a su vez un índice hídrico de $200 \%$.

\section{MARCO TEÓRICO}

La investigación se realiza a nivel de microcuenca, pues se reconoce la importancia de la cuenca hidrográfica como unidad de planificación. Una cuenca es una unidad espacial que está delimitada de manera natural por divisorias de agua y está conformada por una serie de cauces que drenan hacia un cauce principal, se divide en subcuencas y microcuencas. La subcuenca está delimitada por la divisoria de agua de un afluente. La microcuenca es una parte de la subcuenca. (Sheng, 1992) (Ramakrishna, 1997) (Jiménez, 2001).

Las tendencias modernas en materia de legislación de aguas aconsejan incluir expresamente la necesidad de formular e implementar Planes Maestros para la gestión integrada de los recursos hídricos en el ámbito de la región o grupos de cuencas, tomando como unidad de planificación las cuencas hidrográficas. (CEPAL, 1998).

Dado lo anterior se da la importancia de la realización de balances hídricos en cuencas. El balance hídrico es fundamental, debido a que mediante este instrumento se analiza la relación entre variables socioeconómicas como el uso del suelo, y variables naturales como el tipo de suelo, la cantidad de precipitación y la temperatura. Este balance se utiliza en la evaluación de los recursos hídricos con el fin de resolver problemas hídricos, tanto teóricos como prácticos. Por lo tanto, permite evaluar en forma cuantitativa el recurso hídrico, a nivel temporal y espacial. (UNESCO, 2007). 
Mediante el balance hídrico se cuantifican los parámetros involucrados en el ciclo hidrológico, así como los consumos de agua de los diferentes sectores de usuarios, en un área determinada, cuenca, y la interrelación entre ellos. De esta cuantificación resulta un diagnóstico de las condiciones reales del recurso hídrico en cuanto a su oferta, disponibilidad y demanda en dicha área. Debido a que de la aplicación del Balance Hídrico se obtiene un diagnóstico de las condiciones reales del recurso hídrico en un área en particular, es posible tomar medidas y establecer lineamientos y estrategias para su protección y utilización de una manera integrada, garantizando su disponibilidad tanto en cantidad como en calidad (SNET, 2005).

\section{MARCO METODOLÓGICO}

\subsection{BALANCE HÍDRICO}

Partiendo del conocimiento de las precipitaciones medias mensuales y de la evapotranspiración mensual estimada, se puede estudiar el balance del agua en el suelo, a lo largo del año. Conocer el balance de humedad en el suelo, la densidad aparente, la capacidad de campo y el punto de marchitez permanente es importante para evaluar la disponibilidad de agua para los cultivos, los estudios hidrológicos, de conservación de suelos, de drenaje, de repoblación forestal, o el establecimiento del régimen de humedad de los suelos o de criterios de diferenciación climática.

Existen varios modelos para estimar el balance de agua en el suelo; aquí se seguirá el método directo propuesto por Thorthwaite y Mather (1957), según el cual se va perdiendo agua para poder generar la evapotranspiración potencial hasta agotar la reserva.

Según Marini y Piccolo (2000), esta metodología posee dos ventajas importantes:

1) Los datos necesarios para realizar los cálculos (precipitación y temperatura) son de obtención relativamente sencilla,

2) Utiliza valores exclusivamente climáticos para expresar el valor relativo de la precipitación.

En este caso, el balance hídrico se calcula según el uso del suelo, la unidad geomorfológica y la zona climática.

De manera detallada, el procedimiento es el siguiente: (Hernando, L, 1988)

Como primer paso es necesario obtener los valores de precipitación media mensual (P), los cuales deben corregirse eliminando la cantidad que se pierde de escorrentía (Es), así mismo se obtiene como resultado la precipitación corregida (Pc). Posteriormente se estima la evapotranspiración mensual (ETP) con el método de Hargreaves.

Dada la precipitación media mensual y la evapotranspiración, se determina la diferencia mensual entre precipitación y evapotranspiración potencial (Pc-ETP), si los valores resultantes son positivos, la precipitación excede la evapotranspiración 
potencial, y si los valores son negativos, la precipitación no satisface las necesidades meteorológicas.

La pérdida potencial acumulada (PPA), de agua por mes, se obtiene sumando los valores negativos de Pc - ETP a la diferencia mensual.

Además es necesario contar con los valores de la humedad del suelo disponible (HSD) actual por mes, determinada por la capacidad de carga, punto de marchites permanente y profundidad de las raíces, si los valores de Pc - ETP son positivos, mientras que si estos son negativos se estima por la pérdida potencia acumulada de la siguiente manera:

Donde:

$$
\text { HSD: LAD * }\left(\mathrm{e}^{-\left(\mathrm{PPA}^{* A}\right)}\right)
$$

HSD: Humedad del suelo disponible ( $\mathrm{mm})$

LAD: Lámina de agua disponible $=\left((\mathrm{cc}-\mathrm{PMP})^{*}\right.$ Pro $) / 10 \mathrm{~mm}$

Cc: Capacidad de campo (\%)

Pro: Profundidad de las raíces $(\mathrm{cm})$

PPA: Pérdida potencial acumulada (mm)

Posteriormente se calcula el Cambio de la Humedad del Suelo Disponible ( $\Delta \mathrm{HSD}$ ). Se obtiene de la diferencia de la HSD de un mes a otro. Si los valores que se obtienen como resultado son positivos existe una recarga del recurso hídrico y si los datos son negativos es la cantidad de agua cedida a las plantas.

Después, se calcula la evapotranspiración actual (ETA) que se obtiene como el resultado de Pc - ETP, si los valores son positivos la ETA = ETP, y si los valores son negativos la ETA $=\mathrm{Pc}+\Delta \mathrm{HSD}$.

Luego se calcula el Déficit de la humedad del suelo (D), obtenido como el resultado de la diferencia de la ETP - ETA. Y la Ganancia de humedad (G), entendida como el agua que percola hacia las capas inferiores de la siguiente manera: G: HSD del mes anterior + PC - ETP del mes que interesa y restando a ese producto la HSD del mismo interés.

Por último se calcula la Recarga Mensual dada por: $\mathrm{G}$ de c/mes * el área del cultivo analizado.

\subsection{ZONIFICACIÓN}

La zonificación se determinó por medio de la identificación de las áreas de disponibilidad del recurso hídrico, las cuales se determinaron de acuerdo con la metodología empleada por el Instituto de Hidrología, Meteorología y Estudios Ambientales de Colombia (IDEAM), 2004, la cual fue modificada y adaptada para la microcuenca del río Bermúdez en Costa Rica.

Con el objeto de realizar la zonificación, se procedió a calcular la oferta y la demanda hídrica, únicamente considerando la vegetación. De esta manera, se 
considera que la oferta y la demanda depende de las actividades agrícolas o forestales, luego de esta relación se obtiene la disponibilidad hídrica para las poblaciones.

De acuerdo con los resultados del Balance Hídrico, se desarrollaron coberturas espaciales de la humedad del suelo disponible (HSD) (oferta hídrica) y del cambio de humedad del suelo disponible $\triangle \mathrm{HSD}$ (demanda hídrica).

Se calculan los índices de disponibilidad hídrica (Índice de escasez) (IDEAM, 2004), relacionando la oferta hídrica y la demanda hídrica de la siguiente manera:

$$
\mathrm{Ie}=\frac{\triangle \text { HSD (demanda) }}{\text { HSD (oferta) }} * 100
$$

Estos índices se agrupan en cuatro categorías que se describen en el cuadro $\mathrm{N}^{\mathrm{o}} 1$.

Cuadro 1. Índice de Escasez.

\begin{tabular}{|c|c|c|c|}
\hline $\begin{array}{c}\text { Categoría del } \\
\text { índice de escasez }\end{array}$ & $\begin{array}{c}\text { \% de la Oferta } \\
\text { Hídrica utilizada }\end{array}$ & Explicación & Color \\
\hline Alto & $>$ de $40 \%$ & Fuerte presión & Rojo \\
\hline Medio & $20-40 \%$ & $\begin{array}{c}\text { Ordenamiento } \\
\text { Oferta/Demanda }\end{array}$ & Naranja \\
\hline Moderado & $10-20 \%$ & Factor limitador del desarrollo & Amarillo \\
\hline Bajo & $<$ de $10 \%$ & $\begin{array}{c}\text { No se experimentan presiones } \\
\text { importantes sobre el Recurso } \\
\text { Hídrico }\end{array}$ & Verde \\
\hline
\end{tabular}

Fuente: IDEAM, 2004.

Los valores resultantes se representan de forma espacial, dando origen a la zonificación de la disponibilidad del recurso hídrico, tal como se muestra en el cuadro $\mathrm{N}^{\mathrm{o}} 2$ :

Cuadro 2. Disponibilidad Hídrica, según el Índice de Escasez.

\begin{tabular}{|c|c|c|}
\hline $\begin{array}{c}\text { Categoría del índice de } \\
\text { escasez }\end{array}$ & Disponibilidad Hídrica & Color \\
\hline Alto & Baja & Rojo \\
\hline Medio & Moderado & Naranja \\
\hline Moderado & Medio & Amarillo \\
\hline Bajo & Alta & Verde \\
\hline
\end{tabular}

Fuente: IDEAM, 2004. 


\subsection{CONFLICTOS DE USO}

Se realizó un análisis de los conflictos de uso de suelo para determinar la proporción de usos conformes y las áreas que poseen un sobreuso para su posterior análisis, esto se realizó mediante la sobreposición del mapa de uso de suelo con el mapa de capacidad de uso de suelo, el primero de ellos se realizó con base en información del Plan Regional Urbano del Gran Área Metropolitana (PRUGAM), 2008, y con giras de campo para su actualización; el segundo fue elaborado con base en los datos de capacidad de uso de suelo que posee la Fundación Neotrópica, 1998, para las distintas hojas topográficas del país a escala 1: 50000.

Además con estos datos se elaboraron mapas de población y vivienda; finalmente se determinó la oferta y la demanda del recurso hídrico en la microcuenca para con ello obtener la disponibilidad hídrica misma que se obtuvo de acuerdo a los resultados del balance hídrico.

\section{RESULTADOS Y DISCUSIÓN}

\subsection{USO DEL SUELO}

En la microcuenca se encuentran los siguientes usos: bosque, pasto, café, urbano y terrenos descubiertos. A continuación se detallan algunos aspectos acerca de los usos mencionados (Figura 4).

Figura 4. Usos del suelo.

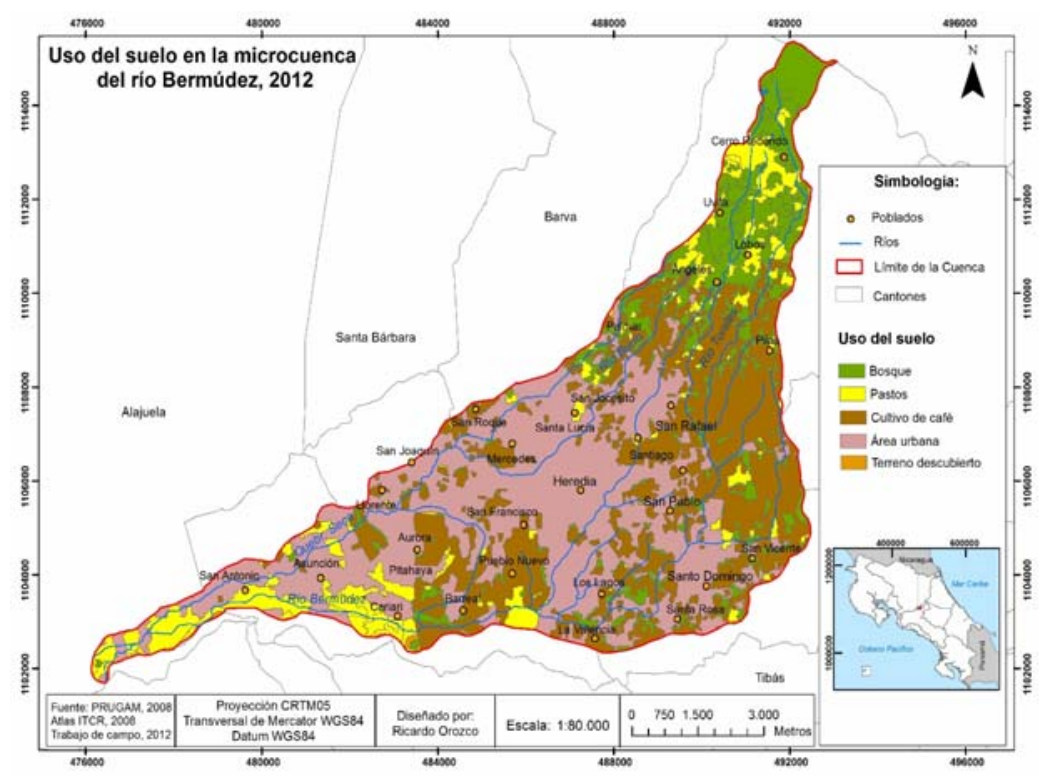

Fuente: Elaboración propia. 


\subsection{BOSQUE}

Esta categoría cubre los sectores de bosque primario y algunos sectores de bosque secundario. El bosque alcanza una extensión de $11.24 \mathrm{Km}^{2}$ es decir, un $15.18 \%$ del área total de la microcuenca.

La mayor extensión de esta cobertura está ubicada a partir de los $2100 \mathrm{msnm}$ en la parte alta de la microcuenca y en el sector del Parque Nacional Braulio Carrillo.

\subsection{PASTO}

Los pastos se han clasificado incluyendo pasto, pasto con árboles y charrales y tacotales. Abarcan una extensión de $8.02 \mathrm{Km}^{2}$, es decir, un $10.83 \%$ de la microcuenca. Se ubican principalmente en dos sectores, el primer sector, en la parte baja de la microcuenca hasta los $1000 \mathrm{msnm}$, cerca del poblado de Asunción; el segundo sector, desde los 1500 msnm hasta colindar con el bosque del Parque Nacional Braulio Carrillo.

\subsection{CAFÉ}

Este cultivo abarca un área de $24.24 \mathrm{Km}^{2}$, correspondiente a un $32.73 \%$ de la microcuenca, siendo el segundo en cobertura en la misma.

Los suelos para cultivo del café deben ser de origen volcánico y con buen drenaje, características presentes en la microcuenca.

El café se da principalmente en los 1000 y 1500 msnm, ubicado mayormente en las comunidades de Santiago, San Rafael, Ángeles, Pilas, Palmar, Pueblo Nuevo y Barreal.

\subsection{URBANO}

Este es el uso más extenso, cubre $30.12 \mathrm{Km}^{2}$, es decir, el $40.67 \%$ de toda la superficie de la microcuenca.

Se localiza en la sección media y baja de la microcuenca, en esta se encuentran centros urbanos importantes como San Rafael, Heredia, San Pablo, San Francisco, Santo Domingo, La Valencia, Los Lagos, Barreal, Mercedes, Santa Lucía, San Roque, San Joaquín, Aurora, Llorente, Cariari, Asunción y San Antonio.

\subsection{TERRENOS DESCUBIERTOS}

Por su ubicación estas áreas podrían ser terrenos en preparación para el cultivo de café. Cubren un área de $0.16 \%$ de la microcuenca $\left(0.12 \mathrm{Km}^{2}\right)$.

De acuerdo a los usos de suelo es importante observar que el área urbana ha ganado terreno, lo cual puede dificultar la disponibilidad hídrica que existe en la microcuenca, ya que la demanda sube pero la oferta podría disminuir, esta situación afecta actualmente durante la época seca en este sector. 
La Figura 5, muestra el crecimiento poblacional que se ha experimentado en la microcuenca de acuerdo a los censos de población del año 1973, 1984, 2000 y 2011. El gráfico representa la realidad en la microcuenca, la cual posee una importante área urbana en crecimiento.

Figura 5. Crecimiento poblacional.

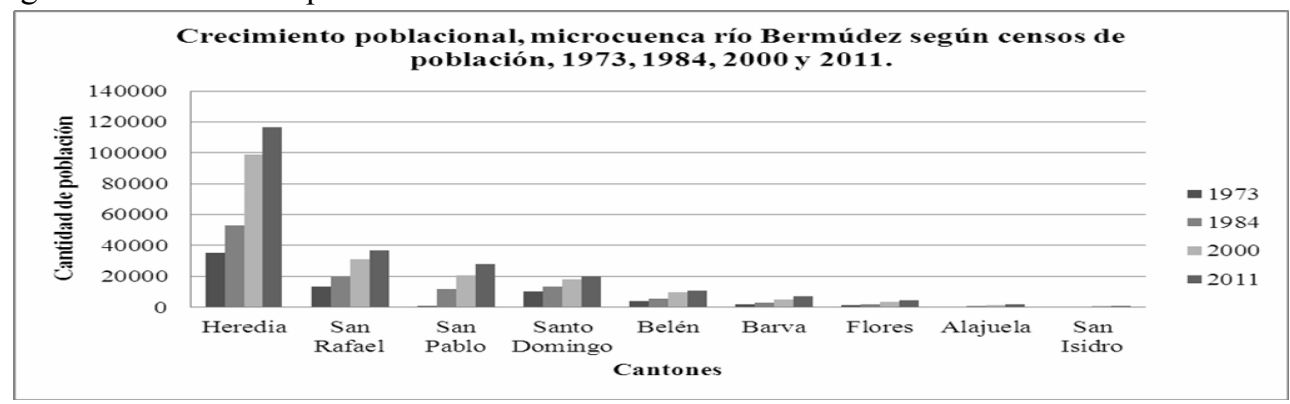

Fuente: Elaboración propia.

Por otra parte, es importante destacar que en la microcuenca se ubican zonas industriales donde trabajan empresas que ejercen presión sobre el recurso hídrico. De acuerdo a PRUGAM, 2008, en esta microcuenca se encuentran 142 empresas aproximadamente, concentradas en la parte central y sur de la misma, en los distritos más urbanos que además cuentan con la mayor cantidad de población y han tenido un crecimiento de la misma en los últimos 35 años (Figura 6).

Figura 6. Actividad industrial.

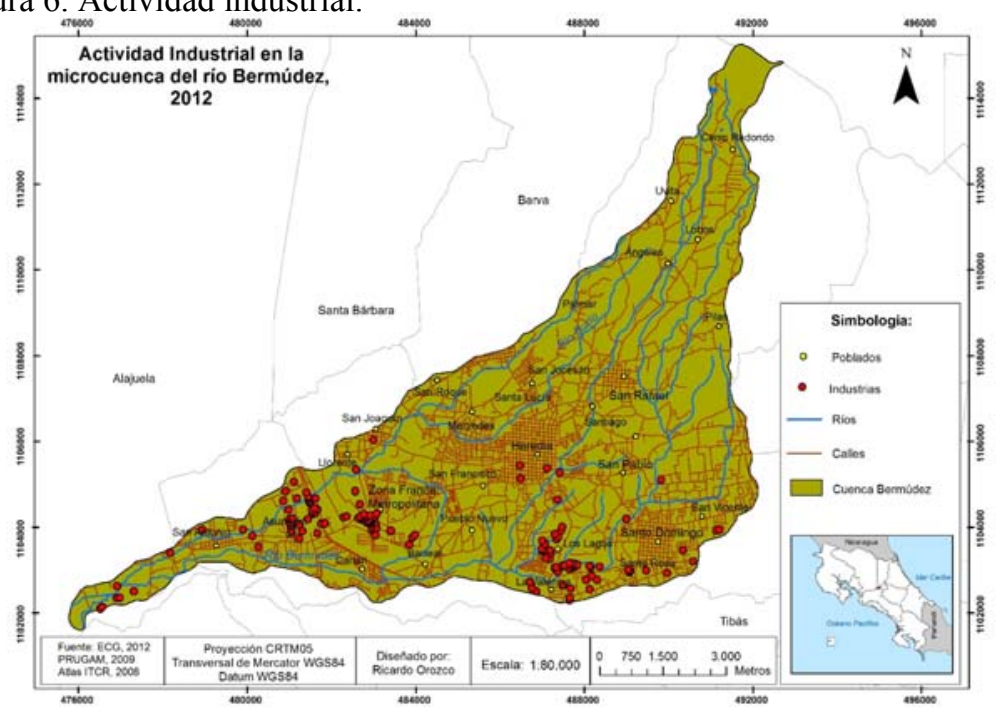

Fuente: Elaboración propia. 
En la zona ubicada al suroeste de la microcuenca se ubican más de 100 industrias, que están dedicadas a distintas actividades, por ejemplo, algunas se dedican al equipo médico, otras a productos sanitarios, maquinaria y aparatos eléctricos, y plásticos y textiles (Cuadro 3).

Cuadro 3. Categorización de industrias ubicadas en microcuenca de río Bermúdez.

\begin{tabular}{|c|c|}
\hline Principales productos & Categoría \\
\hline Manufactura de cuero & $\begin{array}{c}\text { Curtido y Adobo de Cueros; } \\
\text { Fabricación de Maletas. }\end{array}$ \\
\hline $\begin{array}{l}\text { Mayonesa, salsas, galletas, } \\
\text { frutas, procesamiento de carnes y bebidas }\end{array}$ & $\begin{array}{l}\text { Elaboración de Productos Alimenticios } \\
\text { y Bebidas. }\end{array}$ \\
\hline Equipo Médico & $\begin{array}{l}\text { Fabricación de Instrumentos Médicos } \\
\text { y de precisión. }\end{array}$ \\
\hline $\begin{array}{l}\text { Conductores y componentes } \\
\text { eléctricos, circuitos integrados y trans- } \\
\text { formadores }\end{array}$ & $\begin{array}{l}\text { Fabricación de Maquinaria } \\
\text { y Aparatos Eléctricos N.C.P. }\end{array}$ \\
\hline Metalmecánica & Fabricación de Metales Comunes. \\
\hline Papel higiénico, pañales, cartón & Fabricación de Papel y de Productos de Papel. \\
\hline $\begin{array}{c}\text { Bolsas plásticas, tubos rígidos, moldes, } \\
\text { implantes de silicón }\end{array}$ & Fabricación de Productos de Caucho y Plástico. \\
\hline $\begin{array}{l}\text { Textiles, hilos industriales, ropa } \\
\text { íntima }\end{array}$ & Fabricación de Productos Textiles. \\
\hline $\begin{array}{l}\text { Sueros, productos sanitarios y } \\
\text { veterinarios, esteorofón y ropa }\end{array}$ & $\begin{array}{l}\text { Fabricación de Sustancias } \\
\text { y de Productos Químicos. }\end{array}$ \\
\hline
\end{tabular}

Fuente: PRUGAM, 2008.

\subsection{OFERTA}

La alta oferta de recurso hídrico en la microcuenca se ubica en las áreas cubiertas por bosque, principalmente en la unidad geomorfológica volcánica, y abarca 11.24 $\mathrm{Km}^{2}(15.18 \%)$. La oferta media se distribuye por las dos unidades geomorfológicas, en áreas de café, constituyendo el $32.73 \%$ de toda la microcuenca. En lo que se refiere a la baja oferta de recurso hídrico, se encuentra en ambas unidades geomorfológicas en sectores cubiertos por pasto, cubriendo $8.02 \mathrm{Km}^{2}(10.83 \%)$. 


\subsection{DEMANDA}

La demanda alta se encuentra en las áreas cubiertas de bosque, en $11.25 \mathrm{Km}^{2}$ (15.18\%). La media se encuentra en la unidad geomorfológica Sedimentaria, en 23.05 $\mathrm{Km}^{2}(31.12 \%)$. La baja se encuentra en áreas de café de la unidad geomorfológica Volcánica y en las áreas de pasto en toda la microcuenca, cubriendo $9.21 \mathrm{Km}^{2}$ (12.44\%).

\subsection{DISPONIBILIDAD HÍDRICA}

\subsubsection{DISPONIBILIDAD HÍDRICA ALTA}

En la Figura 7 se observa alta disponibilidad de recuso hídrico en las áreas de bosque y café de la unidad geomorfológica Volcánica y en la zonas climáticas III y $\mathrm{IV}$, donde las precipitaciones son las más altas de la microcuenca, siendo en promedio de $3028.52 \mathrm{~mm}$ al año. En estas áreas se presentan también los valores más altos de ganancia, desde $1721.83 \mathrm{~mm}$ hasta $1781.18 \mathrm{~mm}$ al año. La alta disponibilidad se encuentra en el $11.05 \%$ de la microcuenca (Cuadro 4).

Figura 7. Disponibilidad Hídrica

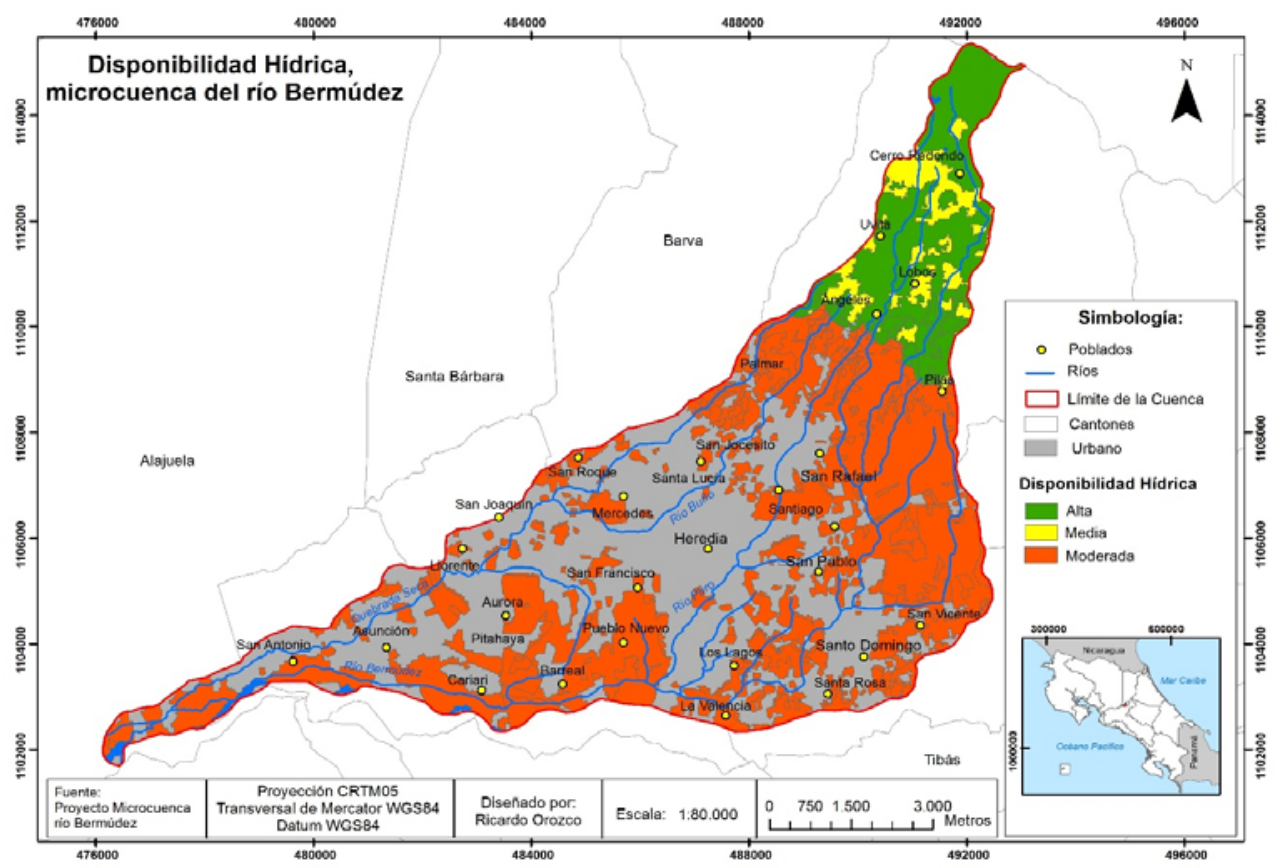

Fuente: Elaboración propia. 


\subsubsection{DISPONIBILIDAD HÍDRICA MEDIA}

En la Figura 7 se puede apreciar que en la microcuenca del río Bermúdez se observa disponibilidad hídrica media en las áreas de pasto, en la unidad geomorfológica Volcánica y zona climática III. Es decir, donde la precipitación es de $3028.52 \mathrm{~mm}$ al año. Esta disponibilidad cubre $2.49 \mathrm{Km}^{2}$, es decir, el $3.36 \%$ de la microcuenca (Cuadro $\mathrm{N}^{\circ}$ 4). Son áreas de transición entre la disponibilidad alta y la moderada. Se evidencia la existencia de la presión del crecimiento urbano.

En estas áreas debería limitarse el desarrollo debido a la reducción de la disponibilidad hídrica (IDEAM, 2005).

Cuadro 4. Disponibilidad del Recurso Hídrico, en $\mathrm{Km}^{2}$ y en porcentaje (2013).

\begin{tabular}{|c|c|c|}
\hline Disponibilidad & $\mathbf{K m}^{\mathbf{2}}$ & Porcentaje \\
\hline Alta & 8.01 & 11.05 \\
\hline Media & 2.49 & 3.36 \\
\hline Moderada & 32.83 & 44.33 \\
\hline
\end{tabular}

Fuente: Elaboración propia.

\subsubsection{DISPONIBILIDAD HÍDRICA MODERADA}

Las áreas de moderada disponibilidad hídrica se encuentran cerca de las zonas urbanas y algunos pequeños sectores limitantes de las áreas de media disponibilidad. Cubren un $44.33 \%\left(32.83 \mathrm{Km}^{2}\right)$ de la microcuenca (Cuadro $\mathrm{N}^{\circ}$ 4). Se ubican en áreas de alto déficit, que oscilan entre $300.46 \mathrm{~mm} / \mathrm{año}$ y $497.55 \mathrm{~mm} / \mathrm{año}$, en la unidad geomorfológica Sedimentaria y en las zonas climáticas I y II. Las precipitaciones son entre $1957.7 \mathrm{~mm}$ /año hasta $2415.62 \mathrm{~mm} /$ año.

En estas áreas debe de existir un ordenamiento de la oferta y la demanda (IDEAM, 2005), pues el recurso hídrico en cualquier momento puede reducir su disponibilidad debido a la presión que existe sobre él. Resulta evidente que debe ocuparse de manera controlada, pues estas áreas cubren casi la mitad de la microcuenca.

Las áreas de media disponibilidad del recurso hídrico corresponden al uso de suelo cubierto por pasto, en la unidad geomorfológica Volcánica y en las zonas climáticas III y IV.

La alta disponibilidad tiene relación con la geomorfología (Mapa $\mathrm{N}^{\circ}$ 2), pero está estrechamente relacionada también con las características climáticas, principalmente la precipitación. Se ubica en la unidad volcánica, y en áreas de mayor precipitación. Además el uso del suelo es bosque $\left(11.34 \mathrm{Km}^{2}\right)$ y café $\left(1 \mathrm{Km}^{2}\right)$ que aunado con la disponibilidad de agua según cultivo, propicia la alta disponibilidad. 
En las áreas de bosque se dan los datos de mayor demanda y mayor oferta, y además son las áreas de mayor disponibilidad en la microcuenca (media), lo que evidencia que son áreas de una considerable producción hídrica.

Se recomienda que de manera prioritaria exista en las áreas de disponibilidad moderada, una reducción del desarrollo urbano. La ganancia generada en estas áreas es media y baja, lo que indica que el recurso hídrico es frágil, en cuanto a su disponibilidad se deberían tomar medidas urgentes.

Es necesario que los gobiernos locales ubicados en la microcuenca participen en su ordenamiento de manera apremiante, pues de seguir la tendencia de crecimiento urbano y poblacional que se ha dado hasta ahora, es posible que en pocos años se esté en áreas de baja disponibilidad en más del $50 \%$ de la microcuenca, provocando escasez de recursos y la consecuente problemática.

\section{BIBLIOGRAFÍA}

CEPAL. (1998). Informe II Taller de Gerentes de Organismos de Cuenca en América latina y el Caribe. 11 al 3 de diciembre de 1997. Santiago, Chile.

BERGOING, J.P. \& MALAVASSI, V. (1982). Mapa Geomorfológico Hoja Abra, escala 1: 50 000, San José, Costa Rica.

BERGOING, J.P. \& MALAVASSI, V. (1982). Mapa Geomorfológico Hoja Barva, escala 1: 50 000, San José, Costa Rica.

BERGOING, J.P. \& MALAVASSI, V. (1982). Mapa Geomorfológico Hoja Río Grande, escala 1: 50 000, San José, Costa Rica.

FUNDACIÓN NEOTRÓPICA (1998). Estudio, análisis y cartografia de la capacidad de uso de las tierras forestales de Costa Rica. Hojas topográficas 1:50000 de capacidad de uso de la tierra. San José, Costa Rica.

FUNDACIÓN NEOTRÓPICA. (1998). Hoja de capacidad de uso de la tierra Abra, escala 1: 50 000, San José, Costa Rica.

FUNDACIÓN NEOTRÓPICA. (1998). Hoja de capacidad de uso de la tierra Barva, escala 1: 50 000, San José, Costa Rica.

FUNDACIÓN NEOTRÓPICA. (1998). Hoja de capacidad de uso de la tierra Río Grande, escala 1:50 000, San José, Costa Rica.

HERNANDO, L. (1988). Balance Hídrico de la Cuenca del Río Poás. Tesis para obtener el grado de Licenciatura en Geografía con énfasis en Geografía Física. Universidad Nacional. Heredia, Costa Rica.

INSTITUTO DE HIDROLOGÍA, METEOROLOGÍA Y ESTUDIOS AMBIENTALES (IDEAM). (2004). Metodología para el cálculo de Índice de Escasez de Agua Superficial. Bogotá, D.C.

INSTITUTO GEOGRÁFICO NACIONAL. Hoja Topográfica Abra, escala 1: 50 000, San José, Costa Rica.

INSTITUTO GEOGRÁFICO NACIONAL. Hoja Topográfica Barva, escala 1: 50 000, San José, Costa Rica.

INSTITUTO GEOGRÁFICO NACIONAL. Hoja Topográfica Río Grande, escala 1: 50 000, San José, Costa Rica. 
JIMÉNEZ. F. (2001). Conceptos, enfoques y estrategias para el manejo de cuencas hidrográficas. Centro Agronómico Tropical de Investigación y Enseñanza (CATIE). Turrialba, Costa Rica

MARINI, M y PICCOLO, M. (2000). El balance hídrico en la Cuenca del río Quequén Salado, Argentina. En Papeles de Geografia, $N^{\circ} 31 . P p .39-53$

PLAN REGIONAL URBANO DE LA GRAN ÁREA METROPOLITANA DE COSTA RICA 2008-2030 (PRUGAM).) (2008). Tomo IV: Atlas Cartográfico Plan PRUGAM 2008-2030. San José, costa Rica.

RAMAKRISHNA, B. (1997). Estrategias de extensión para el manejo integrado de cuencas hidrográficas: conceptos y experiencias. Instituto Interamericano de Cooperación para la Agricultura (IICA) / Deutsche Gesellschaftfür Technische Zusammenarbeit (GTZ). San José, Costa Rica.

SHENG, T.C. (1992). Manual de campo para la ordenación de cuencas hidrográficas. Organización de las Naciones Unidas para la Agricultura y la Alimentación (FAO).

SNET. (2005). Balance Hídrico Integrado y Dinámico en El Salvador. Componente Evaluación de Recursos Hídricos. El Salvador.

THORNTHWAITE C.W. \&MATHER, J.R. (1957). Instructions and tables for computing potential evapotranspiration and the water balance. Drexel Institute of Climatology. Publitions in Climatology. Vol \# 3. Thrid Printing New Jersey.

UNESCO, 2007. Balance hídrico superficial de Costa Rica. Período: 1970-2002. Documentos Técnicos del PHI-LAC, $\mathrm{N}^{\circ}$ 10.Montevideo, Uruguay. 